Marquette University

e-Publications@Marquette

Biomedical Engineering Faculty Research and

Publications

Biomedical Engineering, Department of

$1-1-2012$

\title{
Estimation of Organ and Effective Dose due to Compton Backscatter Security Scans
}

Michael Hoppe

Marquette University

Taly Gilat Schmidt

Marquette University, tal.gilat-schmidt@marquette.edu

Published version. Medical Physics, Vol. 39, No. 6 (2012): 3396-3403. Permalink. (C) 2012 American Institute of Physics. Used with permission. 


\title{
Estimation of Organ and Effective Dose Due To Compton Backscatter Security Scans
}

\author{
Michael E. Hoppe \\ Department of Biomedical Engineering, Marquette University \\ Milwaukee, WI \\ Taly Gilat-Schmidt \\ Department of Biomedical Engineering, Marquette University \\ Milwaukee, WI
}

\begin{abstract}
Purpose: To estimate organ and effective radiation doses due to backscatter security scanners using Monte Carlo simulations and a voxelized phantom set.
\end{abstract}

Methods: Voxelized phantoms of male and female adults and children were used with theGEANT4 toolkit to simulate a backscatter security scan. The backscatter system was modeled based on specifications available in the literature. The simulations modeled a $50 \mathrm{kVp}$ spectrum with $1.0 \mathrm{~mm}$ aluminum-equivalent filtration and a previously measured exposure of approximately $4.6 \mu \mathrm{R}$ at $30 \mathrm{~cm}$ from the source. Photons and secondary interactions were tracked from the source until they reached zero kinetic energy or exited from the simulation's boundaries. The energy deposited in the phantoms' respective organs was tallied and used to calculate total organ dose and total effective dose for frontal, rear, and full scans with subjects located 30 and $75 \mathrm{~cm}$ from the source. 
Results: For a full screen, all phantoms' total effective doses were below the established $0.25 \mu$ Sv standard, with an estimated maximum total effective dose of $0.07 \mu \mathrm{Sv}$ for full screen of a male child. The estimated maximum organ dose due to a full screen was $1.03 \mu \mathrm{Gy}$, deposited in the adipose tissue of the male child phantom when located $30 \mathrm{~cm}$ from the source. All organ dose estimates had a coefficient of variation of less than $3 \%$ for a frontal scan and less than $11 \%$ for a rear scan.

Conclusions: Backscatter security scanners deposit dose in organs beyond the skin. The effective dose is below recommended standards set by the Health Physics Society (HPS) and the American National Standards Institute (ANSI) assuming the system provides a maximum exposure of approximately $4.6 \mu \mathrm{R}$ at $30 \mathrm{~cm}$.

\section{INTRODUCTION}

X-ray backscatter and millimeter wave imaging systems are currently used by the Transportation Security Administration (TSA) for airport passenger and personnel security screening. X-ray backscatter systems use ionizing radiation; whereas millimeter systems use high frequency radio waves. These systems detect radiation scattered by the subject to generate low resolution images intended to depict high contrast between human anatomy and contraband on the surface of the subject. The scanners were first deployed in 2007 as a secondary screening tool and became a primary screening tool, with increased deployment, in 2010. ${ }^{1}$

The American National Standards Institute (ANSI) and the Health Physics Society (HPS) suggest an effective dose upper limit of $0.1 \mu \mathrm{S} v$ per frontal scan and a reference effective dose of $0.25 \mu \mathrm{S} v$ per full screening (frontal and rear). 2,3 The reference effective dose is calculated by measuring the half-value layer and air kerma of a scanner at the point of maximum exposure at distance no closer than $30 \mathrm{~cm}$ from the beam's exit surface. ${ }^{3}$ In comparison, the average American receives an annual effective dose of approximately $3 \mathrm{mSv} .^{4}$

While the ANSI/HPS standard advises an upper dose limit, consensus has not yet been reached on the radiation dose and risk of backscatter scans. ${ }^{5-10}$ An interagency study by the Food and Drug Administration (FDA) and the TSA estimated an effective dose of $0.0372 \mu \mathrm{Sv}$ for a child and $0.0236 \mu \mathrm{Sv}$ for an adult per frontal scan. ${ }^{11}$ The report used mathematical subject models, Monte Carlo simulations with the software package PCXMC, exposure measurements (9.6 $\mathrm{RR}$ ) obtained using a Rapiscan scanner with backplate and Radcal 10X51800 ionization chamber, and proprietary system specifications. ${ }^{11}$ This study had limitations due to the use of a software package designed to

Medical Physics, Vol. 39, No. 6 (2012): pg. 3396-3403. DOI. This article is (C) American Association of Physicists in Medicine and permission has been granted for this version to appear in e-Publications@Marquette. American Association of Physicists in Medicine does not grant permission for this article to be further copied/distributed or hosted elsewhere without the express permission from American Association of Physicists in Medicine. 
simulate medical x-ray systems with an inverse-square divergence, which is not necessarily representative of the system being investigated. ${ }^{11,12} \mathrm{~A}$ study at Johns Hopkins commissioned by the TSA estimated an effective dose of $0.0155 \mu S v$ for an adult due to a full screening using physical exposure measurements $(4.6 \mu \mathrm{R})$ of a dualunit (master and slave scanners) Rapiscan scanner with a Radcal 10X1800 ionization chamber, and previously published conversion factors for converting air kerma and half-value layer values to a reference effective dose per ANSI N43-2009. ${ }^{13}$

Scanner specifications and operation details are of limited availability in the public domain. Access to physical scanners for measurements and assessments is also limited. It should also be noted that the fast scan time and relatively low-energy spectrum introduce uncertainties in the exposure measurements obtained on traditional equipment. ${ }^{8,14}$ To circumvent these limitations, a previous study used image quality metrics, such as resolution and contrast, to estimate a lower bound for the number of detected $x$-rays per detector pixel required to generate images similar to those published from the scanner. ${ }^{7}$ A full screening (frontal and rear) effective dose of $0.88 \mu \mathrm{Sv}$ (3.5 times the ANSI N43.17-2009 standard) was estimated assuming this estimated number of detected photons, the known spectrum, and published $\mathrm{x}$-ray fluence-dose relationships.

Estimating the risk of backscatter scans is also challenging, as the effects of low-dose radiation are not well understood. 5,6,8,9 Mathematical models of risk assume whole body irradiation, whereas backscatter systems are expected to deposit radiation primarily in superficial organs/regions. While there is consensus that the risk to an individual passenger is likely small, the risk to the population may be a concern due to the expected $750 \times 10^{6}$ passenger enplanements in U.S. airports in 2012, increasing to an expected $1 \times 10^{9}$ enplanements by $2030 .{ }^{15}$ However, estimates of population risk rely on estimates of individual risk which further depend on dose estimates. Therefore, difficulties in estimating risk are compounded by uncertainties in the amount and distribution of dose by backscatter scans.

This study estimated the organ and effective dose due to ionizing radiation of backscatter scanners in adults and children using Monte Carlo simulations of radiation transport. Unlike previous studies that relied on mathematical anatomical models and exposure-dose relationships, this study used more anatomically realistic voxelized models representing a broader range of the population. ${ }^{11,13}$ 
NOT THE PUBLISHED VERSION; this is the author's final, peer-reviewed manuscript. The published version may be accessed by following the link in the citation at the bottom of the page.

\section{Materials and Methods}

\section{II.A. Voxelized phantoms}

Four members of the Virtual Family voxelized phantoms representing a 34-yr-old male, a 26-yr-old female, an 11-yr-old female, and a 6-yr-old male were selected to represent a range of the general population. ${ }^{16}$ The selected phantoms have a voxel resolution of $2 \mathrm{~mm}$ in each dimension. The initial segmentations were simplified to include 30 or 31 materials (the adult female included glandular breast tissue, thus an additional material) to decrease the amount of virtual memory and computation required for a simulation. For example, the original segmentations consisted of the skull and the mandible as two distinct materials. These two were simplified to one material, hard bone. Tissue compositions were taken from the International Commission on Radiation Protection Report 110, with the exception of glandular breast tissue which was modeled based on results of Woodard and White. ${ }^{17,18}$ The skeletal regions in the phantoms were segmented into regions of red bone marrow surrounded by hard bone. The breast region was segmented into regions of glandular tissues surrounded by adipose tissue, representing a relatively dense breast.

\section{II.B. Simulation}

A backscatter security scan procedure was simulated using the Monte Carlo radiation transport toolkit, GEANT4 (version 9.3). ${ }^{19}$ The simulations modeled the backscatter system procured by the TSA (Rapiscan Secure 1000, OSI Systems, Hawthorne, CA), using assumptions based on industry standards, scientific, and patent literature. ${ }^{1-3,10-12,21}$ A $50 \mathrm{kVp}$ spectrum with $1.0 \mathrm{~mm}$ aluminumequivalent filtration was modeled using the SPEC78 software. ${ }^{20}$ The aluminum-equivalent filtration thickness of $1.0 \mathrm{~mm}$ was chosen as it is the minimum amount of aluminum-equivalent filtration per the ANSI N43.17-2009 standard. ${ }^{3}$ The simulations modeled a point $x$-ray source with the cone-beam confined to irradiate a $6-\mathrm{mm}$ (height) $\times 100-\mathrm{cm}$ (width) area on the $200 \mathrm{~cm}$ (height) by $100 \mathrm{~cm}$ (width) scan plane located $75 \mathrm{~cm}$ from the source. ${ }^{21}$ In practice, for each vertical position of the source, collimators scan in the horizontal direction to limit the irradiated area for detection purposes. ${ }^{21}$ In the current study, the collimators were not simulated; thus, the complete cone-beam irradiated the subject for each vertical position of the source. The source was translated in the vertical direction to discrete positions separated by $6 \mathrm{~mm}$ (i.e., the vertical extent of the beam at $75 \mathrm{~cm}$ form the source), as suggested by the patent literature. ${ }^{21}$ In this 
configuration, the translated beams are contiguous at the plane $75 \mathrm{~cm}$ from the source (i.e., the expected position of the subject) but are separated by a $0.24 \mathrm{~cm}$ gap in the vertical direction between adjacent beams at $30 \mathrm{~cm}$ from the source, which is the distance where exposure measurements were simulated, per ANSI N43.17-2009. ${ }^{3}$ Conversely, this model results in beam overlap at distances greater than $75 \mathrm{~cm}$ from the source.

The modeled beam provided reduced exposure toward the edges of the scan plane in the horizontal direction, due to the divergence of the fan beam, which corresponds with physical exposure measurements. ${ }^{11}$ Because the simulated configuration translated the source position in the vertical direction, and because of the small beam divergence in the vertical direction (half cone angle of $0.23^{\circ}$ ), the exposure was nearly constant across the vertical direction at the scan plane ( $75 \mathrm{~cm}$ from the source). Another embodiment of the scanner design tilts in the vertical direction rather translating vertically. ${ }^{11}$ Previous exposure measurements quantified a decrease in exposure with vertical distance from the center position, although the decrease in exposure was less than that predicted by the inverse-square law. In the current study, the vertically translating source model was chosen to provide conservative dose estimates.

Our study aimed to simulate realistic scanner exposure. The exposures of the Rapiscan 1000 for a frontal and rear scan were previously measured to be 4.574 and $4.606 \mu R$, respectively $(\sim 0.04$ $\mu G y$ air kerma) in the Johns Hopkins/TSA study that used a horizontal chamber orientation. ${ }^{3,12}$ In order to provide radiation dose estimates that reflect realistic scanner output and conditions, Monte Carlo simulations of the modeled backscatter system were performed to calculate the photon fluence that provided an exposure approximately equal to the previously measured values. In other words, the same source configuration and translation was modeled in the exposure simulations as in the phantom simulations. In the exposure simulations, an $1800 \mathrm{~cm}^{3}$ cylinder of water with a $0.32 \mathrm{~cm}$ polycarbonate shell modeled the Radcal 10X-1800 (Radcal Corp., Monrovia, CA) chamber used in the physical exposure measurements in the FDA/TSA study and the Johns Hopkins/TSA study. As in the Hopkins/TSA study, the simulated chamber was centered $30 \mathrm{~cm}$ from the source per ANSI N43.17-2009 and oriented horizontally. ${ }^{3}$ Different exposure values $(9.6 \mu \mathrm{R})$ were obtained in the FDA/TSA study when the chamber was placed in the vertical orientation (chamber axis perpendicular to the beam), due to the larger irradiated surface area. ${ }^{11,12}$ The horizontal orientation was selected in the current study because the Hopkins/TSA exposure measurements are those officially

Medical Physics, Vol. 39, No. 6 (2012): pg. 3396-3403. DOI. This article is (C) American Association of Physicists in Medicine and permission has been granted for this version to appear in e-Publications@Marquette. American Association of Physicists in Medicine does not grant permission for this article to be further copied/distributed or hosted elsewhere without the express permission from American Association of Physicists in Medicine. 
made public by the TSA. The chamber was simulated as a water cylinder instead of air due to the low number of photon interactions in air. Air kerma was calculated by scaling the dose in water by an ffactor of 0.971 .22 The number of photons was adjusted until the simulations produced exposure estimates equal to the measured values.

Based on results of the exposure simulations, $3.6 \times 10^{6}$ photons were simulated per cone-beam (area of $6000 \mathrm{~mm}^{2}$ at a distance of 75 $\mathrm{cm}$ ) for all phantom simulations. Dose is expected to depend on subject position relative to the source. The ANSI N43.17-2009 standard states that $30 \mathrm{~cm}$ is the minimum distance from the source at which an exposure measurement can be performed. ${ }^{3}$ The patent literature suggests that the subject is positioned $75 \mathrm{~cm}$ from the source. ${ }^{21}$ Based on these suggested distances, our study estimated organ doses with the central coronal plane of the phantom located 30 and $75 \mathrm{~cm}$ from the plane on which the source is located. X-ray photons and secondary particles were tracked until absorption or exit from the phantom. The simulations tallied the energy, in electronvolts $(\mathrm{eV})$, deposited in each voxel.

\section{II.C. Dose estimates}

Organ dose was calculated in units of grays (Gy) by dividing the energy deposited in an organ by the organ mass. The mean, $\mu$, standard deviation, $\sigma$, and percent coefficient of variation ( $\% \mathrm{COV}$ ), $100 \% \times \sigma / \mu$, of the organ doses were calculated across three independent simulations of each phantom. Three simulations were performed to estimate the \%COV, thus allowing an estimate of the uncertainty of the dose estimates. Due to the computational nature of the full-body Monte Carlo simulations, there is a tradeoff between the number of simulations per phantom and the uncertainty in the dose estimates. In this work, numerous factors contribute to the uncertainty of the dose estimates in addition to the Monte Carlo simulations, as will be discussed in Sec. IV. Three trials per phantom was considered an adequate tradeoff between computation time and uncertainty. Radiation dose estimates for a full screening were equal to the sum of the frontal-scan and rear-scan dose estimates.

The effective dose due to a full screen (frontal and rear) for each phantom was also calculated similarly to the method described in ICRP Publication 103, as expressed in Eq. (1), where radiation quality factor, Q, equals one for x-ray Compton backscatter and the tissue specific weighting factors, $\mathrm{W}$, are listed in Table $\mathrm{I}^{23}$ 
NOT THE PUBLISHED VERSION; this is the author's final, peer-reviewed manuscript. The published version may be accessed by following the link in the citation at the bottom of the page.

$$
\text { Effective dose }=\sum_{T} Q \cdot \text { Dose }_{T} \cdot W_{T} .
$$

The voxelized phantoms did not contain all organs required by the effective dose calculation. Due to this limitation, dose to the salivary glands, oral mucosa, extrathoracic region, and lymphatic nodes were estimated as described in ANSI N43.17-2009. ${ }^{3}$ Also, the female child's thyroid organ dose was used when calculating the male child's effective dose due to its absence in the male child phantom.

TABLE I. Tissue weighting factors for calculating effective dose.

\begin{tabular}{lc}
\hline \hline Tissue & Weighting factor $\left(W_{T}\right)$ \\
\hline $\begin{array}{l}\text { Red marrow, colon, lung, stomach, } \\
\text { breast, remainder tissues }\end{array}$ & 0.12 \\
Gonads & \\
Bladder, esophagus, liver, thyroid & 0.08 \\
Bone surface, brain, skin & 0.04 \\
\hline \hline
\end{tabular}

${ }^{a}$ Remainder tissues: adrenals, extrathoracic region, gall bladder, heart, kidneys, lymphatic nodes, muscle, oral mucosa, pancreas, prostate, small intestine, spleen, thymus, and uterus/cervix.

\section{RESULTS}

Tables II-V display the mean organ dose estimates for all phantoms for frontal and rear scans. The coefficient of variation was less than $2.7 \%$ for all organ doses from a frontal scan at both distances (30 and $75 \mathrm{~cm}$ ). For the rear scan, the coefficient of variation was $10.3 \%$ for the adult female's eye lens at $75 \mathrm{~cm}, 7.1 \%$ for the adult male's eye lens at $75 \mathrm{~cm}, 5.2 \%$ for the male child's eye lens, and below 3\% for all other organs of all phantoms at both distances. Table VI lists the effective doses for frontal scans, rear scans, and full screens estimated from the organ doses at 30 and $75 \mathrm{~cm}$. 
NOT THE PUBLISHED VERSION; this is the author's final, peer-reviewed manuscript. The published version may be accessed by following the link in the citation at the bottom of the page.

TARe II. Mean $(n=3)$ argan $(3 x e$ estmates for a fromal san at $30 \mathrm{~cm}$.

\begin{tabular}{|c|c|c|c|c|}
\hline Drgm & Adadt male (Gy) & Adalt femaile (Gy) & Male child (Oy) & Fennole child (Gy) \\
\hline Bene: & $8.87 \times 10^{-4}$ & $9.80 \times 10^{-4}$ & $1.72 \times 10^{-7}$ & $1.21 \times 10^{-7}$ \\
\hline Cartilage & $\operatorname{tin}=10^{-7}$ & $6.87 \times 19^{-4}$ & $1.79 \times 10^{-t}$ & $794 \times 10^{-4}$ \\
\hline Skin & $7.48 \times 10^{-4}$ & $8.35 \times 10^{-6}$ & $9.54 \times 10^{-6}$ & $8.46 \times 10^{-8}$ \\
\hline Hloced & $2.00 \times 10^{-k}$ & $3.19 \times 10^{-6}$ & $3.06 \times 10^{-6}$ & $3.50 \times 10^{-8}$ \\
\hline Mascile & $3.45 \times 10^{-1}$ & $3.63 \times 10^{-8}$ & $5.20 \times 10^{-1}$ & $4.57 \times 10^{-7}$ \\
\hline Sort tissae & $9.56 \times 10^{-17}$ & $2.20 \times 10^{-30}$ & $3,01 \times 10^{-1}$ & $1.12 \times 10^{-7}$ \\
\hline Adipose & $2.95 \times 10^{-7}$ & $3.65 \times 10^{-7}$ & $4.25 \times 10^{-7}$ & $3.76 \times 10^{-7}$ \\
\hline Lug & $2.46 \times 10^{-4}$ & $2.65 \times 10^{-1}$ & $4.15 \times 10^{-1}$ & $3 / 2 \times 10^{-4}$ \\
\hline Contuective tissue & $3,46 \times 10^{-4}$ & $2.48 \times 10^{-6}$ & $4.33 \times 10^{-6}$ & $3.08 \times 10^{-8}$ \\
\hline Eye lens & $1.33 \times 10^{-7}$ & $132 \times 10^{-7}$ & $1.16 \times 10^{-1}$ & $9.87 \times 10^{-4}$ \\
\hline Stomach & $4.24 \times 10^{-1}$ & $4.33 \times 10^{-6}$ & $5.12 \times 10^{-1}$ & $6.31 \times 10^{-x}$ \\
\hline Testesfovary & $1.15 \times 10^{-7}$ & $1.92 \times 10^{-4}$ & $1.24 \times 10^{-7}$ & $3.15 \times 10^{-4}$ \\
\hline Adrenal & $8.57 \times 10^{-7}$ & $9.23 \times 10^{-9}$ & $1.53 \times 10^{-1}$ & $1.47 \times 10^{-1}$ \\
\hline Galli hlanddes & $3,41 \times 10^{-6}$ & $3.72 \times 10^{-6}$ & $4.96 \times 10^{-8}$ & $3.07 \times 10^{-8}$ \\
\hline Hear: & $3.08=10^{-4}$ & $3.15 \times 10^{-k}$ & $4.7 \pi \times 10^{-4}$ & $4.21 \times 10^{-4}$ \\
\hline Pancreas & $2.78 \times 10^{-1}$ & $3.92 \times 10^{-6}$ & $4,11 \times 10^{-8}$ & $6.74 \times 10^{-3}$ \\
\hline Spleren & $6.83=10^{-6}$ & $137 \times 10^{-6}$ & $2.41 \times 10^{-6}$ & $2.37 \times 10^{-4}$ \\
\hline Thyroid: & $1.57 \times 10^{-7}$ & $1.04 \times 10^{-7}$ & $N / A^{2}$ & $1.01 \times 10^{-2}$ \\
\hline Btadider & $3,96 \times 10^{-1}$ & $5.55 \times 10^{-6}$ & $6.22 \times 10^{-1}$ & $4,4 \mathrm{E} \times 10^{-3}$ \\
\hline Kitney & $1.06 \times 10^{-1}$ & $1.90 \times 10^{-k}$ & $2.94 \times 10^{-1}$ & $2.31 \times 10^{-4}$ \\
\hline Intestine & $2.90 \times 10^{-6}$ & $3.54 \times 10^{-6}$ & $9.79 \times 10^{-9}$ & $4.47 \times 10^{-3}$ \\
\hline Liver & $3.11 \times 10^{-6}$ & $2.89 \times 10^{-6}$ & $3.82 \times 10^{-4}$ & $3.77 \times 10^{-8}$ \\
\hline Red marrew & $3.45 \times 10^{-1}$ & $5.77 \times 10^{-9}$ & $1.23 \times 10^{-1}$ & $655 \times 10^{-7}$ \\
\hline Colon & $4.28 \times 10^{-1}$ & $5.00 \times 10^{-6}$ & $7.76 \times 10^{-1}$ & $5.65 \times 10^{-6}$ \\
\hline Brain & $2.29 \times 10^{-4}$ & $1.61 \times 10^{-9}$ & $7,60 \times 10^{-9}$ & $6.09 \times 10^{-4}$ \\
\hline Esopitazus & $3.47 \times 10^{-2}$ & $3.02 \times 10^{-6}$ & $2,90 \times 10^{-1}$ & $2,77 \times 10^{-7}$ \\
\hline Prostatefutents & $1,13 \times 10^{-6}$ & $1.79 \times 10^{-6}$ & $2.17 \times 10^{-4}$ & $2.81 \times 11^{-4}$ \\
\hline Thymus & $2.64 \times 10^{-1}$ & $3.96 \times 10^{-6}$ & $6.07 \times 10^{-4}$ & $3.58 \times 10^{-8}$ \\
\hline Breas & N/A & $6.91 \times 10^{-6}$ & $N / A$ & N/A \\
\hline
\end{tabular}

Thyroed gland absert from this phattioen.

Thaiz III. Meazi $(\mathrm{E}-3)$ organ dose estimates for a raut scan at $30 \mathrm{~cm}$

\begin{tabular}{|c|c|c|c|c|}
\hline Orzant & Adult male (Gy) & Aduil fenale (Gy) & Male ctrild (Gy) & Female chald (Gy) \\
\hline Boces & $9.26 \times 10^{-5}$ & $1.06 \times 10^{-7}$ & $2.06 \times 10^{-7}$ & $1.31 \times 10^{-7}$ \\
\hline Cantalage & $3.14 \times 10^{-5}$ & $2.78 \times 16^{-1}$ & $3,74 \times 10^{-3}$. & $4.17 \times 10^{-6}$ \\
\hline Sain & $7.88 \times 10^{-5}$ & $8.66 \times 10^{-4}$ & $1.11 \times 10^{-t}$ & $9.49 \times 10^{-6}$ \\
\hline Blood & $3.30 \times 10^{-\rightarrow}$ & $2.35 \times 10^{-4}$ & $4.42 \times 10^{-\rightarrow}$ & $6.51 \times 10^{-9}$. \\
\hline Musete. & $434 \times 10^{-4}$ & $-4.57 \times 10^{-4}$ & $7.26 \times 10^{-4}$ & $557 \times 10^{-6}$ \\
\hline Sodt tissue & $6.13 \times 10^{-9}$ & $2.74 \times 10^{-10}$ & $3.55 \times 10^{-4}$ & $8.85 \times 10^{-9}$ \\
\hline Aitpose & $3.73 \times 10^{-7}$ & $4.79 \times 10^{-7}$ & $6.06 \times 10^{-7}$ & $633 \times 10^{-\gamma}$ \\
\hline Lung & $208 \times 10^{-5}$ & $3.71 \times 10^{-4}$ & $2.75 \times \mathrm{ti}^{-4}$ & $2 \pi \times 10^{-6}$ \\
\hline Coesaertive tissate & $355 \times 10^{-7}$ & $2.57 \times 10^{-4}$ & $5.19 \times 10^{-1}$ & $203 \times 10^{-5}$ \\
\hline Eye lems & $1.45 \times 10^{-15}$ & $2.21 \times 10^{-10}$ & $2.99 \times 10^{-10}$ & $2.74 \times 10^{-20}$ \\
\hline Stomath & $3.77 \times 10^{-9}$ & $8.62 \times 10^{-7}$ & $1.20 \times 10^{-1}$ & $127 \times 10^{-5}$ \\
\hline Tetrsjovary & $3.46 \times 10^{-6}$ & $192 \times 10^{-4}$ & $2.10 \times 10^{-8}$ & $1.22 \times 10^{-6}$ \\
\hline Adrenal & $5.59 \times 10^{-9}$ & $2.76 \times 10^{-1}$ & $157 \times 10^{-4}$ & $2.47 \times 10^{-6}$ \\
\hline Gall blaidiet & $3.80 \times 10^{-9}$ & $5,11 \times 10^{-7}$ & $858 \times 10^{-7}$ & $1.19 \times 10^{-5}$ \\
\hline Hean & $425 \times 10^{-9}$ & $1.00 \times 10^{-4}$ & $6.66 \times 10^{-7}$ & $869 \times 10^{-9}$ \\
\hline Pancreas: & $4.91 \times 10^{-9}$ & $1.56 \times 10^{-4}$ & $1,16 \times 10^{-4}$ & $380 \times 10^{-9}$ \\
\hline Spueent & $2.16 \times 10^{-4}$ & $4.43 \times 10^{-4}$ & $3.79 \times 10^{-3}$ & $4.49 \times 10^{-6}$ \\
\hline niyzaid & $1.90 \times 10^{-9}$ & $3.97 \times 10^{-4}$ & $\mathrm{~N} / \mathrm{A}^{2}$ & $3.11 \times 10^{-9}$ \\
\hline Masder & $107 \times 10^{-5}$ & $1.08 \times 10^{-4}$ & $2.59 \times 10^{-3}$ & $1.73 \times 10^{-8}$ \\
\hline Kidtiey. & $2.23 \times 10^{-6}$ & $3,54 \times 16^{-4}$ & $5.09 \times 10^{-1}$ & $450 \times 10^{-8}$ \\
\hline Intrestion & $2.98 \times 10^{-9}$. & $4.23 \times 10^{-9}$ & $2.73 \times 10^{-7}$ & $4.66 \times 10^{-9}$ \\
\hline Livet & $9.27 \times 10^{-7}$ & $1.98 \times 10^{-4}$ & $2.08 \times 10^{-3}$ & $1.71 \times 10^{-4}$ \\
\hline Red narroos & $3.10 \times 10^{-9}$ & $3.00 \times 10^{-4}$ & $9.92 \times 10^{-4}$ & $505 \times 10^{-9}$ \\
\hline Calon & $1.19 \times 10^{-5}$ & $8.60 \times 10^{-1}$ & $2.04 \times 10^{-3}$ & $200 \times 10^{-6}$. \\
\hline Brain & $4.48 \times 10^{-9}$ & $3.55 \times 10^{-1}$ & $9.89=10^{-7}$ & $8.29 \times 10^{-9}$ \\
\hline Esopingus & $3.43 \times 10^{-}$? & $5.25 \times 10^{-3}$ & $588 \times 10^{-7}$ & $4.16 \times 10^{-9}$ \\
\hline Proctatefatienus & $2.90 \times 10^{-4}$ & $1.71 \times 10^{-4}$ & $4.34 \times 10^{-1}$ & $1.62 \times 10^{-6}$ \\
\hline Thynus & $6.70 \times 10^{-30}$ & $2.83 \times 10^{-4}$ & $4.20 \times 10^{-3}$ & $3.75 \times 10^{-4}$ \\
\hline Breast: & NiA & $5.90 \times 10^{-9}$ & NiA & N/A \\
\hline
\end{tabular}

Thyroid gland absent from fius piantom.

Medical Physics, Vol. 39, No. 6 (2012): pg. 3396-3403. DOI. This article is C American Association of Physicists in Medicine and permission has been granted for this version to appear in e-Publications@Marquette. American Association of Physicists in Medicine does not grant permission for this article to be further copied/distributed or hosted elsewhere without the express permission from American Association of Physicists in Medicine. 
NOT THE PUBLISHED VERSION; this is the author's final, peer-reviewed manuscript. The published version may be accessed by following the link in the citation at the bottom of the page.

TAmie IV Mam in $=3$ ) argan dose estimates for a frontal san at $75 \mathrm{~cm}$

\begin{tabular}{|c|c|c|c|c|}
\hline Organ & Adalt male (Gy) & Adult female (Gy) & Male chald . Fy) & Female chuld (Gy) \\
\hline Bases & $4.92 \times 10^{-8}$ & $3.29 \times 10^{-3}$ & $8.73 \times 10^{-8}$ & $6.52 \times 10^{-1}$ \\
\hline Cantilage & $5.15 \times 10^{-6}$ & $3.55 \times 10^{-5}$ & $8.38 \times 10^{-5}$ & $4.07 \times 10^{-8}$ \\
\hline Skin & $4.21 \times 10^{-5}$ & $4.53 \times 10^{-1}$ & $4,78 \times 10^{-7}$ & $4,40 \times 10^{-1}$ \\
\hline Blood & $1.00 \times 10^{-6}$ & $1.65 \times 10^{-4}$ & $1.74 \times 10^{-3}$ & $1.71 \times 10^{-1}$ \\
\hline Muscie. & $2.02 \times 10^{-5}$ & $2.03 \times 10^{-4}$ & $2.76 \times 10^{-4}$ & $2.45 \times 10^{-5}$ \\
\hline Sodt tissue & $4.63 \times 10^{-9}$ & $109 \times 10^{-10}$ & $1.50 \times 10^{-9}$ & $5.54 \times 10^{-7}$ \\
\hline Adipose & $1.61 \times 10^{-2}$ & $2.04 \times 10^{-2}$ & $221 \times 10^{-7}$ & $1.99 \times 10^{-3}$ \\
\hline Lare & $1.28 \times 10^{-6}$ & $1: 39 \times 10^{-3}$ & $2.12 \times 10^{-7}$ & $1.82 \times 10^{-1}$ \\
\hline Comective tissas & $1.70=10^{-6}$ & $1.31 \times 10^{-4}$ & $2.17 \times 10^{-4}$ & $1.62 \times 10^{-5}$ \\
\hline Eyeless & $597 \times 10^{-5}$ & $5.64 \times 10^{-4}$ & $4.69 \times 10^{-4}$ & $5.03 \times 10^{-5}$ \\
\hline Scomact & $202 \times 10^{-6}$ & $2.13 \times 10^{-3}$ & $2.55 \times 10^{-8}$ & $3.63 \times 10^{-1}$ \\
\hline Testes/ovary & $5.36 \times 10^{-4}$ & $9.57 \times 10^{-4}$ & $5.89 \times 10^{-4}$ & $1.65 \times 10^{-1}$ \\
\hline Adretal & $434 \times 10^{-9}$ & $5.13 \times 10^{-9}$ & $8.36 \times 10^{-7}$ & $8.63 \times 10^{-9}$ \\
\hline Giell bladiler & $167 \times 10^{-6}$ & $1.88 \times 10^{-8}$ & $2.45 \times 10^{-8}$ & $1.65 \times 10^{-6}$ \\
\hline Beat & $1.51 \times 10^{-5}$ & $1.56 \times 10^{-4}$ & $2.29 \times 10^{-4}$ & $2.12 \times 10^{-5}$ \\
\hline Pattueas & $134 \times 10^{-4}$ & $1.97 \times 10^{-3}$ & $204 \times 10^{-3}$ & $3.31 \times 10^{-4}$ \\
\hline Spleen & $4.84 \times 10^{-9}$ & $7.91 \times 10^{-9}$ & $132 \times 10^{-4}$ & $1.25 \times 10^{-1}$ \\
\hline Thyroid & $702 \times 10^{-6}$ & $5.00 \times 10^{-8}$ & N/A & $4.92 \times 10^{-1}$ \\
\hline Hacter & $1.50=10^{-8}$ & $2.64 \times 10^{-8}$ & $315 \times 10^{-8}$ & $2.32 \times 10^{-1}$ \\
\hline Kidtiey: & $5.94 \times 10^{-9}$ & $1.05 \times 10^{-5}$ & $1.54 \times 10^{-4}$ & $1.26 \times 10^{-5}$ \\
\hline Intrestixe & $142 \times 10^{-7}$ & $1,72 \times 10^{-4}$ & $492 \times 10^{-7}$ & $2.17 \times 10^{-1}$ \\
\hline Liver & $1.53 \times 10^{-4}$ & $1.09 \times 10^{-4}$ & $1.89 \times 10^{-4}$ & $1.98 \times 10^{-1}$ \\
\hline Reć marrow & $1.75 \times 10^{-9}$ & $1.52 \times 10^{-9}$ & $5.96 \times 10^{-9}$ & $3.11 \times 10^{-3}$ \\
\hline Colant & $214 \times 10^{-8}$ & $3.21 \times 10^{-9}$ & $3.78 \div 10^{-8}$ & $2.97 \times 10^{-1}$ \\
\hline Brain & $108 \times 10^{-9}$ & $2.54 \times 10^{-5}$ & $253 \times 10^{-9}$ & $2.91 \times 10^{-7}$ \\
\hline Esopitagis & $162 \times 10^{-5}$ & $7.84 \times 10^{-3 b}$ & $1.43 \times 10^{-4}$ & $1.44 \times 10^{-5}$ \\
\hline Prookatehationu & $5.20 \times 10^{-9}$ & $1.53 \times 10^{-4}$ & $101 \times 10^{-1}$ & $1.49 \times 10^{-1}$ \\
\hline Thynas & $1.17 \times 10^{-4}$ & $9.37 \times 10^{-9}$ & $2.86 \times 10^{-2}$ & $1.82 \times 10^{-5}$ \\
\hline Hreast & $\mathrm{N} / \mathrm{A}$ & $3.65 \times 10^{-8}$ & N/A & $\mathrm{N} / \mathrm{A}$ \\
\hline
\end{tabular}

Thyroid glanil absers from stuis plantom.

Tuarn V. Mean (n - 3) argat dose escimates for a rear scan at $75 \mathrm{~cm}$

\begin{tabular}{|c|c|c|c|c|}
\hline Orgin & Atalt male (Gy) & Adalt female (Oy) & Male craid (Gy) & Femake chibs (Gy) \\
\hline Hoce & $4.24 \times 10^{-8}$ & $437 \times 10^{-1}$ & $8.67 \times 10^{-4}$ & $5.31 \times 10^{-8}$ \\
\hline Cartlage & $1.24 \times 10^{-6}$ & $1.16 \times 10^{-1}$ & $1.52 \times 10^{-3}$ & $1.73 \times 10^{-5}$ \\
\hline Shin & $3.82 \times 10^{-8}$ & $398 \times 10^{-1}$ & $4.13 \times 10^{-t}$ & $4 \times 1 \times 10^{-5}$ \\
\hline Bloos & $1.90 \times 10^{-9}$ & $101 \times 10^{-1}$ & $2.02 \times 10^{\circ}$ & $2.58 \times 10^{-9}$ \\
\hline Muscle & $2.06 \times 10^{-6}$ & $200 \times 10^{-1}$ & $2.78 \times 10^{-4}$ & $2.4 \times 10^{-6}$ \\
\hline Soft tissue & $2.58 \times 10^{-3}$ & $9.68 \times 10^{-11}$ & $1.28 \times 10^{-7}$ & $3.65 \times 10^{-7}$ \\
\hline Adipose & $1.68 \times 10^{-7}$ & $226 \times 10^{-7}$ & $2.24 \times 10^{-7}$ & $2.51 \times 10^{-7}$ \\
\hline Lumg & $7,96 \times 10^{-9}$ & $139 \times 10^{-7}$ & $1.02 \times 10^{-2}$ & $1.01 \times 10^{-5}$ \\
\hline Cocnective Tisur & $1.30 \times 10^{-6}$ & $990 \times 10^{-1}$ & $1.52 \times 30^{-4}$ & T.64 $\times 10^{-3}$ \\
\hline Eyelens & $124 \times 10^{-13}$ & $1.84 \times 10^{-10}$ & $235 \times 10^{-10}$ & $2.19 \times 10^{-20}$ \\
\hline Stomach & $1.95 \times 10^{-6}$ & $386 \times 10^{-7}$ & $5.15 \times 10^{-4}$ & $5.06 \times 10^{9}$ \\
\hline Testesblovary & $1.35 \times 10^{-4}$ & $753 \times 10^{-7}$ & $8.49 \times 10^{-7}$ & $4,79 \times 10^{9}$ \\
\hline Adremal & $2.38 \times 10^{-4}$ & $9.95 \times 10^{-7}$ & $6.64 \times 10^{-4}$ & $9.61 \times 10^{-4}$ \\
\hline Gall bladder & $1.84 \times 10^{-6}$ & $2.25 \times 10^{-4}$ & $3.49 \times 10^{-1}$ & $5.19 \times 10^{-7}$ \\
\hline Houn: & $2.36 \times 10^{-9}$ & $467 \times 10^{-1}$ & $3,34 \times 10^{-4}$ & $3.49 \times 10^{-9}$ \\
\hline Pancreas & $1.87 \times 10^{-9}$ & $6.87 \times 10^{-7}$ & $4.05 \times 10^{-7}$ & $1,80 \times 10^{3}$ \\
\hline Splem & $1.19 \times 10^{-8}$ & $182=10^{-1}$ & $1.60=10^{-1}$ & $1.61 \times 10^{-5}$ \\
\hline Thyroid. & $961 \times 10^{-27}$ & $1.76 \times 10^{-1}$ & $\mathrm{~N}^{\prime} \mathrm{A}^{*}$ & $1,39 \times 10^{-4}$ \\
\hline Bladier & $3.94 \times 10^{-9}$ & $394 \times 10^{-7}$ & $9.64 \times 10^{-7}$ & $6.28 \times 10^{3}$ \\
\hline Kidney & $9.45 \times 10^{-9}$ & $1.43 \times 10^{-1}$ & $1.88 \times 10^{-1}$ & $160 \times 10^{-1}$ \\
\hline Dresine & $1.42 \times 19^{-9}$ & $1.82 \times 10^{-7}$ & $1.15 \times 10^{-1}$ & $200 \times 10=$ \\
\hline Liver & $4.22 \times 10^{-9}$ & $842 \times 10^{-7}$ & $1.98 \times 10^{-1}$ & $733 \times 10^{-3}$ \\
\hline Red marsow & $1.27 \times 10^{-9}$ & $1.70 \times 10^{-7}$ & $8.01 \times 10^{-7}$ & $2.27 \times 10^{-7}$ \\
\hline Cobos & $5.17 \times 10^{-9}$ & $3.24 \times 10^{-7}$ & $3.77 \times 10^{-4}$ & $8.14 \times 10^{-9}$ \\
\hline Bnin & $1.70 \times 10^{-9}$ & $1.47 \times 10^{-7}$ & $8.31 \times 10^{-1}$ & $3,22 \times 10^{-9}$ \\
\hline Esopitazis & $1.48 \times 10^{-9}$ & $217 \times 10^{-4}$ & $4.09 \times 10^{-4}$ & $1.60 \times 10^{-4}$ \\
\hline Prostatefuterus & $9.83 \times 10^{-9}$ & $6.14 \times 10^{-4}$ & $2.40 \times 10^{-7}$ & $5,49 \times 10^{-3}$ \\
\hline Thymus & $337 \times 10^{\text {s }}$ & $120 \times 10^{-4}$ & $1.61 \times 10^{-4}$ & $1.55 \times 10^{-9}$ \\
\hline Besest & N/A & $170 \times 10^{-t}$ & $\mathrm{~N} / \mathrm{A}$ & NA \\
\hline
\end{tabular}

Tayrod glased absem from this phamtom

Medical Physics, Vol. 39, No. 6 (2012): pg. 3396-3403. DOI. This article is (C) American Association of Physicists in Medicine and permission has been granted for this version to appear in e-Publications@Marquette. American Association of Physicists in Medicine does not grant permission for this article to be further copied/distributed or hosted elsewhere without the express permission from American Association of Physicists in Medicine. 
NOT THE PUBLISHED VERSION; this is the author's final, peer-reviewed manuscript. The published version may be accessed by following the link in the citation at the bottom of the page.

\begin{tabular}{|c|c|c|c|c|c|c|}
\hline Phactim & Frostal scas $(30 \mathrm{~cm})$ & Fromtal scial $(75 \mathrm{~cm})$ & Rear scaut $(30 \mathrm{~cm})$ & Rear sean (75 cm) & Full screen (30 cm) & Full screen $(75 \mathrm{~cm}$ ) \\
\hline Male adalt & 0.64 & 0.02 & 0.01 & 0.01 & 005 & 0.93 \\
\hline Fermale adalt & 0.04 & 0.02 & 0.02 & 0.01 & 006 & 005 \\
\hline Mato criald & 005 & 002 & 0,02 & 0.01 & $00 \%$ & $O A A$ \\
\hline Ferale cuiles & 0.64 & 0.92 & 0.12 & 0.01 . & 006 & 0.33 \\
\hline
\end{tabular}

\section{Discussion}

The purpose of our study was to estimate organ dose resulting from Compton backscatter security scanners to enable more accurate estimates of risk. The effective dose was determined from the estimated organ doses. The ANSI standard states the effective does shall not exceed $0.10 \mu \mathrm{Sv}$ for a frontal scan and $0.25 \mu \mathrm{Sv}$ for a full screening. All four phantoms received less than the recommended effective dose limits. The maximum effective dose for a frontal scan was $0.05 \mu \mathrm{Sv}$ (male child), which is about $50 \%$ of the recommended effective dose limit for a frontal scan. The maximum effective dose for a full screen was $0.07 \mu \mathrm{Sv}$ (male child), which is approximately $30 \%$ of the recommended full screen effective dose limit. The dose performance of the scanners with respect to the frontal dose limit may be more meaningful in terms of risk than the full screening limit, as the frontal scans generally irradiate more radiosensitive organs (i.e., eye lens and thyroid). For example, the eye lens had a maximum frontal scan mean dose of $1.33 \times 10^{-7} \mathrm{~Gy}$ and a maximum rear scan mean dose of $2.99 \times 10^{-10} \mathrm{~Gy}$, a ratio of approximately $444: 1$. Both maximum effective dose values previously stated occurred with the phantom centered $30 \mathrm{~cm}$ from the source. As seen is Table VI, effective dose decreased by roughly a factor of two when the phantom was positioned at $75 \mathrm{~cm}$ from the source compared to $30 \mathrm{~cm}$. Overall, results at the 30 and $75 \mathrm{~cm}$ phantom positions provide dose estimates at a range of expected subject positions.

Including all phantoms at both distances, individual total organ doses ranged from $2.06 \times 10^{-10} \mathrm{~Gy}$ (soft tissue of the adult female at $75 \mathrm{~cm}$ ) to $1.03 \times 10^{-6} \mathrm{~Gy}$ (adipose of the male child at $30 \mathrm{~cm}$ ) for a full screen. To put these numbers in perspective, the breast, skin, and eye lens dose from a full screen of an adult female was $0.09,0.21$, and $0.14 \mu \mathrm{Gy}$, respectively, compared to 4500,504 , and $2.9 \mu \mathrm{Gy}$ for a two-view mammogram of one breast ( $25 \mathrm{kVp}, \mathrm{Mo} / \mathrm{Mo}) .{ }^{24}$ Thus, the organ doses from the backscatter scan are at least 1 order of magnitude and as much as 5 orders of magnitude lower than a twoview mammography study. For further comparison, a maximum total effective dose of $0.07 \mu \mathrm{Sv}$ from a full screening is comparable to the dose due to cosmic radiation during about one minute, at $3.9 \mu \mathrm{Sv} / \mathrm{h}$, of

Medical Physics, Vol. 39, No. 6 (2012): pg. 3396-3403. DOI. This article is @ American Association of Physicists in Medicine and permission has been granted for this version to appear in e-Publications@Marquette. American Association of Physicists in Medicine does not grant permission for this article to be further copied/distributed or hosted elsewhere without the express permission from American Association of Physicists in Medicine. 
flight between New York, NY and Chicago, IL. ${ }^{25}$ However, it may be difficult to draw conclusions from this comparison, as the type and distribution (i.e., organ dose) differ between the two types of exposure.

The effective dose values estimated in the current study for a full screen at $75 \mathrm{~cm}$ were approximately two times higher than those estimated in the study commissioned by the TSA, performed by Johns Hopkins Applied Physics Lab. ${ }^{13}$ This difference may be due to the fact that the TSA/Johns Hopkins study used half-value layer and exposure measurements to dose conversion factors rather than system specific simulations with voxelized phantoms. The effective dose values estimated for a frontal scan at $75 \mathrm{~cm}$ were approximately a factor of two lower for an adult, when compared to estimates by the FDA/TSA report, while the dose to a child was similar. ${ }^{11}$ This may be due to differences in the simplified mathematical phantoms compared to the current voxelized phantoms, limitations of the PCXMC software package, and potential differences in the studied scanner models. ${ }^{11}$ Table VII displays the studies which rely on radiation exposure measurements for determining dose estimates. The effective dose estimated in the current study is approximately an order of magnitude lower than that previously estimated by a study that estimated the photon fluence by analyzing published images. ${ }^{7}$ While estimating the photon fluence from the published images eliminates the dependence on possibly inaccurate exposure measurements, additional issues may arise due to unknown image processing routines performed by the systems.

\begin{tabular}{|c|c|c|c|c|c|c|c|}
\hline Srady & Scienert & Exposure Gro & Plathom & Age & Heiehtr (m) & Weiglts (kt) & Effective dose (jSsy) \\
\hline \multirow[t]{2}{*}{ FDATSA } & \multirow[t]{2}{*}{ Simple amit: } & \multirow[t]{2}{*}{9.6 (vertical) } & Mánit & 30 & 1.74 & $\pi$ & $0.0372^{*}$ \\
\hline & & & Cald & 5 & 1.09 & 19.1 & $0.0236^{\prime \prime}$ \\
\hline TSNJotus Hopikins" & Daal-urit & 4.6 (borizumal) & - & - & - & - & $0.015 s^{t}$ \\
\hline \multirow[t]{4}{*}{ Conent study } & \multirow[t]{4}{*}{ Dal-iest } & \multirow[t]{4}{*}{4.6 (locizontal) } & Adalt male & 34 & 1,77 & 72.4 & $0025^{4}$ \\
\hline & & & Acalt female & 26 & 1.6 .7 & 58.7 & $0.028^{t}$ \\
\hline & & & Mule chilas: & 6 & 1.17 & 193 & $0,037^{2}$ \\
\hline & & & Fenaie chald & 11 & 1.47 & 35.4 & $0.027^{2}$ \\
\hline
\end{tabular}

A limitation of the study is that organ doses were calculated for the specific phantom models, and may not be applicable to variations in subject size and anatomy. However, the dose estimates may be useful for calculating risk to typical adult and child subjects. Collimator penumbra is not included in the source modeling. The penumbra 
contributes to the overall photon flux, which is estimated in the current study by matching the simulated and measured exposures.

Furthermore, the presented results are based on numerous assumptions that affect the dose estimates. A simplification of the ionization chamber as a water cylinder encased in a polycarbonate shell was used when calibrating the simulation, whereas the physical equipment likely has more components and possibly more materials. This was done due to the proprietary design of the chamber. Also, the water chamber may introduce additional inaccuracies due to attenuation of the low-energy spectra. It is difficult to determine the impact of this on the results; however, results are relatively in agreement with standards and previously published work (Table VII). Using the vertically translating model instead of the stationary source model introduces error primarily due to the constant and smaller distance between the source and the scan plane as the vertical distance from center increases. Assuming a decrease in exposure according to the inverse-square law, the exposure of the translating source model is approximately 3 times greater at the upper and lower limits of the $200 \mathrm{~cm}$ scan plane compared to the stationary source model. However, a majority of the tissues used to calculate the effective dose are not located in these upper and lower scan plane regions, so this will likely have little effect on the effective dose estimate. When using a stationary source, dose to superior organs, such as the brain and eye lens, depend on an individual's height, with taller subjects receiving a lower dose to superior organs. The vertically translating model source model used in the current work provides conservative dose estimates and may be useful from the perspective of radiation protection.

Another limitation of the current work is that the dose estimates depend on previously measured exposure measurements, which may have limited accuracy. Also, the simulated chamber orientation may also impact dose values. It has been suggested that these published exposure measurements may underestimate the true exposure due to the high dose rate, low-energy spectrum, and limitations of the specific ion chambers. ${ }^{8,13}$ Furthermore, the patent literature, from which the simulated system was modeled, represents a preferred embodiment and may not represent the specifications of the manufactured product currently in use at airports.

Additional investigation is required to provide more accurate dose estimates. Public disclosure of the system specifications would enable more accurate system modeling. Additional exposure measurements must be obtained with ionization chambers that have

Medical Physics, Vol. 39, No. 6 (2012): pg. 3396-3403. DOI. This article is @ American Association of Physicists in Medicine and permission has been granted for this version to appear in e-Publications@Marquette. American Association of Physicists in Medicine does not grant permission for this article to be further copied/distributed or hosted elsewhere without the express permission from American Association of Physicists in Medicine. 
been validated for the low-energy spectra, low exposure, and high flux rate produced by the backscatter scanners. To validate the Monte Carlo models, an additional exposure measurement should be performed in a second phantom and compared to the Monte Carlo predictions. The low-energy spectra and low fluence may make it challenging to design an appropriate validation phantom. Overall, the dose values estimated in the current study are intended to estimate the order of magnitude of the radiation dose, and may require modification as more detailed scanner specifications become available.

\section{Conclusions}

Overall, this paper presents radiation dose estimates from backscatter security scanners for a range of subjects, which may be used for risk estimates and for understanding the impact of backscatter scanners on the average annual effective dose. The estimated doses comply with standards set by ANSI/HPS in N43.142009 for radiation safety for personnel security screening systems using $x$-ray or gamma radiation assuming an exposure of approximately $4.6 \mu \mathrm{R}$ at $30 \mathrm{~cm}$, as previously measured according to the ANSI standard. A maximum total effective dose of $0.0529 \mu$ Sv due to a frontal scan and a maximum total effective dose of $0.0730 \mu \mathrm{Sv}$ due to a full screening are below the 0.1 and $0.25 \mu$ Sv limits. However, as with all imaging modalities using ionizing radiation, the risk must be weighed against the benefit, both of which must be quantified for backscatter security scans.

\section{Acknowledgments}

This research was funded in part by National Science Foundation awards OCI-0923037 "MRI: Acquisition of a Parallel Computing Cluster and Storage for the Marquette University Grid (MUGrid)" and CBET0521602 "Acquisition of a Linux Cluster to Support College-Wide Research \& Teaching Activities." Dr. Lars Olson and David Herzfeld assisted with the use of this resource. Franco Rupcich of Marquette University assisted in validating the simulation software.

\section{References}

1. National Council on Radiation Protection and Measurments, "Screening of humans for security purposes using ionizing radiation scanning systems," NCRP Commentary No. 16 (National Council on Radiation Protection and Measurements, Bethesda, MD, 2003).

Medical Physics, Vol. 39, No. 6 (2012): pg. 3396-3403. DOI. This article is (C) American Association of Physicists in Medicine and permission has been granted for this version to appear in e-Publications@Marquette. American Association of Physicists in Medicine does not grant permission for this article to be further copied/distributed or hosted elsewhere without the express permission from American Association of Physicists in Medicine. 
NOT THE PUBLISHED VERSION; this is the author's final, peer-reviewed manuscript. The published version may be accessed by following the link in the citation at the bottom of the page.

2. American National Standards Institute, Radiation Safety for Personnel Security Screening Systems Using X-rays (Health Physics Society, McLean, VA, 2002).

3. American National Standards Institute, Radiation Safety for Personnel Security Screening Systems Using X-ray or Gamma Radiation (Health Physics Society, McLean, VA, 2009).

4. National Council on Radiation Protection and Measurements, "Ionizing radiation exposure of the population of the United States," NCRP Report No. 160 (NCRP, Bethesda, MD, 1993).

5. D. J. Brenner, "Are x-ray backscatter scanners safe for airport passenger screening? For most individuals, probably yes, but a billion scans per year raises long-term public health concerns," Radiology 259(1), 6-10 (2011).http://dx.doi.org/10.1148/radiol.11102347

6. P. Mehta and R. Smith-Bindman, "Airport full-body screening: What is the risk?," Arch. Intern Med. 171(12), 1112-1115

(2011).http://dx.doi.org/10.1001/archinternmed.2011.105

7. P. Rez, R. L. Metzger, and K. L. Mossman, "The dose from Compton backscatter screening," Radiat. Prot. Dosim. 145(1), 75-81 (2010).http://dx.doi.org/10.1093/rpd/ncq358

8. P. Rez, "Uncertainties in the dose from full-body airport screening," Arch. Intern Med. 171(12), 1129 (2011).http://dx.doi.org/10.1001/archinternmed.2011.258

9. D. A. Schauer, "Does security screening with backscatter x-rays do more good than harm?," Radiology 259(1), 12-16 (2011).http://dx.doi.org/10.1148/radiol.11102376

10. Radiobiology Research Team at the Civil Aerospace Medical Institute, XRay Backscatter Security Scanners at U.S. Airports, Federal Air Surgeon's Medical Bulletin, 49(1) (2011).

11. F. Cerra, Assessment of the Rapiscan Secure $1000^{\circledR}$ Body Scanner for Conformance With Radiological Safety Standards (United States Department of Homeland Security Transportation Security Administration and the United States Food and Drug AdministrationCenter for Devices and Radiological Health, Silver Spring, MD, 2006).

12. M. Tapiovaara, M. Lakkisto, and A. Servomaa, PCXMC: A PC-Based Monte Carlo Program for Calculating Patient Doses in Medical X-ray Examinations (Finnish Centre for Radiation and Nuclear Safety, Helsinki, 1997).

Medical Physics, Vol. 39, No. 6 (2012): pg. 3396-3403. DOI. This article is @ American Association of Physicists in Medicine and permission has been granted for this version to appear in e-Publications@Marquette. American Association of Physicists in Medicine does not grant permission for this article to be further copied/distributed or hosted elsewhere without the express permission from American Association of Physicists in Medicine. 
NOT THE PUBLISHED VERSION; this is the author's final, peer-reviewed manuscript. The published version may be accessed by following the link in the citation at the bottom of the page.

13. Johns Hopkins University Applied Physics Laboratory, "Radiation safety engineering assessment report for the Rapiscan Secure 1000 in single pose," Report No. NSTD-09-1065 version 2.0 (Johns Hopkins University, Laurel, MD, 2010).

14. O. Hupe and U. Ankerhold, "X-ray security scanners for personnel and vehicle control: Dose quantities and dose values," Eur. J. Radiol. 63, 237-241 (2007).http://dx.doi.org/10.1016/j.ejrad.2007.04.029

15. Federal Aviation Administration Aerospace Forecast: Fiscal Years 20102030 (FAA, Washington, DC, 2010).

16. A. Christ, W. Kainz, E. G. Hahn, K. Honegger, M. Zefferer, E. Neufeld, W. Rascher, R. Janka, W. Bautz, and J. Chen, "The Virtual FamilyDevelopment of surface-based anatomical models of two adults and two children for dosimetric simulations," Phys. Med. Biol. 55, N23 (2010).http://dx.doi.org/10.1088/0031-9155/55/2/N01

17. International Commission on Radiological Protection, "Adult reference computational phantoms," Ann. ICRP 39(2), (Pergamon, Oxford, 2009), ICRP Publication No. 110.

18. H. Q. Woodard and D. R. White, "The composition of body tissues," Br. J. Radiol. 59, 1209-1218 (1986).http://dx.doi.org/10.1259/0007-128559-708-1209

19. S. Agostinelli, J. Allison, K. Amako, J. Apostolakis, H. Araujo, P. Arce, M. Asai, D. Axen, S. Banerjee, G. Barrand, F. Behner, L. Bellagamba, J. Boudreau, L. Broglia, A. Brunengo, H. Burkhardt, S. Chauvie, J. Chuma, R. Chytracek, G. Cooperman, G. Cosmo, P. Degtyarenko, A. Dell'Acqua, G. Depaola, D. Dietrich, R. Enami, A. Feliciello, C. Ferguson, H. Fesefeldt, G. Folger, F. Foppiano, A. Forti, S. Garelli, S. Giani, R. Giannitrapani, D. Gibin, J. J. Gómez Cadenas, I. González, G. Gracia Abril, G. Greeniaus, W. Greiner, V. Grichine, A. Grossheim, S. Guatelli, P. Gumplinger, R. Hamatsu, K. Hashimoto, H. Hasui, A. Heikkinen, A. Howard, V. Ivanchenko, A. Johnson, F. W. Jones, J. Kallenbach, N. Kanaya, M. Kawabata, Y. Kawabata, M. Kawaguti, S. Kelner, P. Kent, A. Kimura, T. Kodama, R. Kokoulin, M. Kossov, H. Kurashige, E. Lamanna, T. Lampén, V. Lara, V. Lefebure, F. Lei, M. Liendl, W. Lockman, F. Longo, S. Magni, M. Maire, E. Medernach, K. Minamimoto, P. Mora de Freitas, Y. Morita, K. Murakami, M. Nagamatu, R. Nartallo, P. Nieminen, T. Nishimura, K. Ohtsubo, M. Okamura, S. O’Neale, Y. Oohata, K. Paech, J. Perl, A. Pfeiffer, M. G. Pia, F. Ranjard, A. Rybin, S. Sadilov, E. Di Salvo, G. Santin, T. Sasaki, N. Savvas, Y. Sawada, S. Scherer, S. Sei, V. Sirotenko, D. Smith, N. Starkov, H. Stoecker, J. Sulkimo, M. Takahata, S. Tanaka, E. Tcherniaev, E. Safai Tehrani, M. Tropeano, P. Truscott, H. Uno, L. Urban, P. Urban, M. Verderi, A. Walkden, W. Wander, H. Weber, J. P. Wellisch, T. Wenaus, D. C. Williams, D. Wright, T. Yamada, H. Yoshida, 
NOT THE PUBLISHED VERSION; this is the author's final, peer-reviewed manuscript. The published version may be accessed by following the link in the citation at the bottom of the page.

D. Zschiesche, and GEANT4 Collaboration, "GEANT4-A simulation toolkit," Nucl. Instum. Methods A 506(3), 250-303 (2003).http://dx.doi.org/10.1016/S0168-9002(03)01368-8

20. K. Cranley, B. Gilmore, G. Fogarty, and L. Desponds, "Catalogue of diagnostic X-ray spectra and other data," IPEM Report No. 78.

21. S. W. Smith and IRT Corporation, "X-ray backscatter detection system," U.S. patent 5,181,234 (Jan 19, 1993).

22. J. M. Boone, "The trouble with CTDI," Med. Phys. 34, 1364 (2007).http://dx.doi.org/10.1118/1.2713240

23. A. D. Wrixon, "New ICRP recommendations," J. Radiol. Prot. 28, 161-168 (2008).http://dx.doi.org/10.1088/0952-4746/28/2/R02

24. I. Sechopoulos, S. Suryanarayanan, S. Vedantham, C. J. D'Orsi, and A. Karellas, "Radiation dose to organs and tissues from mammography: Monte Carlo and phantom study," Radiology 246, 434-443 (2008).http://dx.doi.org/10.1148/radiol.2462070256

25. W. Friedberg and K. Copeland, What Aircrews Should Know About Their Occupational Exposure to Ionizing Radiation (Civil Aerospace Medical Institute, FAA, Oklahoma City, OK, 2003).

Author to whom correspondence to be addressed. Electronic mail:

michael.hoppe@marquette.edu; Telephone: (414) 288-4447.

Electronic mail: tal.gilat-schmidt@marquette.edu

Medical Physics, Vol. 39, No. 6 (2012): pg. 3396-3403. DOI. This article is (C) American Association of Physicists in Medicine and permission has been granted for this version to appear in e-Publications@Marquette. American Association of Physicists in Medicine does not grant permission for this article to be further copied/distributed or hosted elsewhere without the express permission from American Association of Physicists in Medicine. 